\title{
Abnormal Breast Detection Via Combination of Particle Swarm Optimization and Biogeography-Based Optimization
}

\author{
Fangyuan Liu ${ }^{1,2, a, *}$, Koji Nakamura ${ }^{3}$, Rodney Payne ${ }^{4}$ \\ ${ }^{1}$ School of Computer Science and Technology, Nanjing Normal University, Nanjing, Jiangsu \\ 210023, China \\ 2Jiangsu Key Laboratory of 3D Printing Equipment and Manufacturing, Nanjing, Jiangsu 210042, \\ China \\ ${ }^{3}$ School of Computer Science and Systems Engineering, Kyushu Institute of Technology, lizuka- \\ shi, Fukuoka Prefecture 820-8502, Japan \\ ${ }^{4} J o h n$ E. Walker Department of Economics, Clemson University, Clemson, SC 29634, USA \\ a2191513453@qq.com
}

Keywords: particle swarm optimization; biogeography-based optimization; abnormal breast; identification; classification; detection.

\begin{abstract}
The breast cancer is the most common cancer among women. To detect it in an accurate way, we designed a new abnormal breast detection system based on the hybridization of particle swarm optimization and biogeography-based optimization. The simulation results showed the sensitivity achieved $87.90 \pm 0.88 \%$, the specificity achieved $87.20 \pm 2.74 \%$, and the accuracy achieved $87.55 \pm 1.34 \%$. Our method is better than two state-of-the-art methods.
\end{abstract}

\section{Background}

The breast cancer is the most common cancer among women in China, USA, and other countries. There are a massive of types of abnormal breast in the early stage of breast cancer. The famous miniMIAS database [1] have collected in total six types: (1) Circumscribed Mass; (2) Asymmetry; (3) Architectural distortion; (4) Calcification; (5) Ill-defined masses; (6) Spiculated masses.

Traditionally, the manual check suffers from inter-expert and intra-expert variance. Besides, the emotion will influence the identification accuracy. Hence, scholars tend to use computer-vision techniques. For example: Liu (2016) [2] proposed a weighted-type fractional Fourier transform. Wu (2016) [3] proposed a chaotic adaptive real-coded biogeography-based optimization. Chen (2016) [4] proposed a two-stage algorithm. In the first stage, they employed wavelet energy entropy (WEE) as the feature. In the second stage, they used the linear regression classifier (LRC) as the classification tool. Abdel-Zaher (2016) [5] used deep belief networks. Magna (2016) [6] employed an ensemble of artificial immune system models.

We analyzed above methods, and found their identification accuracy is low and can not be applied in practical situation. The main reason is their classifier is not trained well. Hence, our team proposes a novel hybrid algorithm, which is a hybridization of particle swarm optimization (PSO) [7] and biogeography-based optimization (BBO) [8].

\section{Materials and Methods}

200 images from the mini-MIAS database [1] were selected. 100 are of abnormal breast image, and the rest 100 are of normal breast image. All the six abnormal types were regarded as one "Abnormal" class. The preprocessing step was used according to reference [3]. It includes the additive and multiplicative noise reduction, image enhancement, background, and pectoral muscle removal.

Two-level wavelet entropy (WE) was used as the features of brain images. The WE is based on a wavelet transform followed by the entropy calculation over the wavelet subbands [9-13]. Haar wavelet was chosen, as it is the most common wavelet used in various fields. 
We used the single-hidden-layer back propagation neural network (BPNN) [14-16] as the classifier. The training of BPNN is not robust, since the initialization will be updated at random for each training. To solve it, scholars have proposed numerous bioinspired algorithms [17, 18]. In this study, we proposed a Hybridization of Particle swarm optimization and Biogeography-based optimization (abbreviated as HPB). The former one mimics the bird swarm [19], while the latter one mimics the migration behavior over islands [20-22]. The core idea is to divide the population into two halves: One performs PSO and the other performs BBO. In each iteration, both PSO and BBO perform searching individually. Nevertheless, in the update, the best of the whole population are selected from the whole population. Our method is different from the HBP algorithm proposed in literature [23].

\section{Experiments and Results}

The 10x10-fold cross validation was used. The sensitivity results are shown in Table 1. The specificity results are shown in Table 2 . The accuracy results are shown in Table 3.

Table 1. Sensitivity Result

\begin{tabular}{|c|c|c|c|c|c|c|c|c|c|c|c|}
\hline & F1 & F2 & F3 & F4 & F5 & F6 & F7 & F8 & F9 & F10 & Total \\
\hline R1 & 90 & 70 & 100 & 90 & 90 & 90 & 80 & 90 & 90 & 90 & 88 \\
\hline R2 & 100 & 100 & 100 & 70 & 90 & 100 & 90 & 70 & 80 & 80 & 88 \\
\hline R3 & 80 & 90 & 90 & 100 & 90 & 90 & 100 & 60 & 80 & 100 & 88 \\
\hline R4 & 90 & 80 & 100 & 90 & 90 & 80 & 100 & 90 & 80 & 90 & 89 \\
\hline R5 & 100 & 90 & 80 & 100 & 80 & 80 & 90 & 100 & 80 & 80 & 88 \\
\hline R6 & 80 & 80 & 100 & 100 & 60 & 90 & 80 & 90 & 90 & 100 & 87 \\
\hline R7 & 80 & 100 & 80 & 80 & 80 & 80 & 100 & 100 & 80 & 100 & 88 \\
\hline R8 & 100 & 90 & 90 & 90 & 90 & 80 & 80 & 80 & 100 & 90 & 89 \\
\hline R9 & 90 & 80 & 80 & 90 & 80 & 70 & 90 & 100 & 100 & 100 & 88 \\
\hline R10 & 100 & 90 & 80 & 70 & 100 & 80 & 100 & 70 & 80 & 90 & 86 \\
\hline Average & & & & & & & & & & & $87.90 \pm 0.88$ \\
\hline
\end{tabular}

\begin{tabular}{|c|c|c|c|c|c|c|c|c|c|c|c|}
\hline \multicolumn{12}{|c|}{ Table 2. Specificity Result } \\
\hline & F1 & $\mathrm{F} 2$ & F3 & $\mathrm{F} 4$ & F5 & F6 & F7 & F8 & F9 & F10 & Total \\
\hline R1 & 70 & 90 & 80 & 100 & 80 & 90 & 90 & 80 & 90 & 90 & 86 \\
\hline R2 & 80 & 100 & 100 & 90 & 90 & 90 & 100 & 90 & 90 & 90 & 92 \\
\hline R3 & 90 & 80 & 90 & 90 & 100 & 90 & 100 & 80 & 80 & 80 & 88 \\
\hline R4 & 90 & 90 & 80 & 70 & 90 & 90 & 100 & 90 & 90 & 80 & 87 \\
\hline R5 & 80 & 90 & 100 & 90 & 90 & 100 & 100 & 70 & 90 & 80 & 89 \\
\hline R6 & 80 & 90 & 90 & 70 & 80 & 80 & 80 & 90 & 90 & 100 & 85 \\
\hline R7 & 80 & 90 & 100 & 90 & 100 & 90 & 100 & 90 & 80 & 70 & 89 \\
\hline R8 & 90 & 90 & 90 & 80 & 90 & 90 & 80 & 60 & 70 & 100 & 84 \\
\hline R9 & 90 & 70 & 100 & 60 & 90 & 70 & 90 & 80 & 90 & 90 & 83 \\
\hline R10 & 100 & 100 & 90 & 90 & 90 & 60 & 100 & 80 & 80 & 100 & 89 \\
\hline Average & & & & & & & & & & & $87.20 \pm 2.74$ \\
\hline \multicolumn{12}{|c|}{ Table 3. Accuracy Result } \\
\hline & F1 & $\mathrm{F} 2$ & F3 & F4 & F5 & F6 & F7 & F8 & F9 & F10 & Total \\
\hline R1 & 80 & 80 & 90 & 95 & 85 & 90 & 85 & 85 & 90 & 90 & 87.0 \\
\hline R2 & 90 & 100 & 100 & 80 & 90 & 95 & 95 & 80 & 85 & 85 & 90.0 \\
\hline R3 & 85 & 85 & 90 & 95 & 95 & 90 & 100 & 70 & 80 & 90 & 88.0 \\
\hline R4 & 90 & 85 & 90 & 80 & 90 & 85 & 100 & 90 & 85 & 85 & 88.0 \\
\hline R5 & 90 & 90 & 90 & 95 & 85 & 90 & 95 & 85 & 85 & 80 & 88.5 \\
\hline R6 & 80 & 85 & 95 & 85 & 70 & 85 & 80 & 90 & 90 & 100 & 86.0 \\
\hline R7 & 80 & 95 & 90 & 85 & 90 & 85 & 100 & 95 & 80 & 85 & 88.5 \\
\hline R8 & 95 & 90 & 90 & 85 & 90 & 85 & 80 & 70 & 85 & 95 & 86.5 \\
\hline R9 & 90 & 75 & 90 & 75 & 85 & 70 & 90 & 90 & 95 & 95 & 85.5 \\
\hline R10 & 100 & 95 & 85 & 80 & 95 & 70 & 100 & 75 & 80 & 95 & 87.5 \\
\hline Average & & & & & & & & & & & $87.55 \pm 1.34$ \\
\hline
\end{tabular}


The sensitivity, specificity, and accuracy over 10x10-fold cross validation are $87.90 \pm 0.88 \%$, $87.20 \pm 2.74 \%$, and $87.55 \pm 1.34 \%$, respectively. In terms of accuracy, our result is better than MIPTPS method [24] and GLCM + SVM [25] as shown in Table 4.

Table 4. Comparison

\begin{tabular}{|c|c|}
\hline Method & Accuracy \\
\hline MIP-TPS [24] & $84.8 \pm 3.1$ \\
\hline GLCM + SVM [25] & 62.0 \\
\hline Our Method & $87.55 \pm 1.34$ \\
\hline
\end{tabular}

In the future, we shall discuss other optimization algorithms, which may serve as training algorithms, such as genetic algorithm [26, 27], artificial bee colony [28-30], bacterial chemotaxis optimization [31, 32], and firefly algorithm [33]. Besides, some variants of SVM shall be tested, including fuzzy SVM [34, 35] and twin SVM [36, 37].

\section{Conclusion and Discussions}

Our team proposed a novel hybridization of PSO and BBO. The experiment result shows its effectiveness. We shall enroll more data in our future studies.

\section{Acknowledgments}

This paper is supported by Open Program of Jiangsu Key Laboratory of 3D Printing Equipment and Manufacturing (3DL201602), Open fund for Jiangsu Key Laboratory of Advanced Manufacturing Technology (HGAMTL1601).

\section{References}

[1] The mini-MIAS database of mammograms. 2016 ; Available from: http: //peipa. essex. ac.uk/info/mias.html.

[2] Liu, G., Computer-aided diagnosis of abnormal breasts in mammogram images by weighted-type fractional Fourier transform. Advances in Mechanical Engineering, 2016. 8(2), Article ID: 11

[3] $\mathrm{Wu}, \mathrm{X}$., Smart detection on abnormal breasts in digital mammography based on contrast-limited adaptive histogram equalization and chaotic adaptive real-coded biogeography-based optimization. Simulation, 2016. 92(9): pp. 873-885

[4] Chen, Y., et al. Wavelet energy entropy and linear regression classifier for detecting abnormal breasts. Multimedia Tools and Applications, 2016, DOI: 10.1007/s11042-016-4161-0 (Online).

[5] Abdel-Zaher, A.M. and A.M. Eldeib, Breast cancer classification using deep belief networks. Expert Systems with Applications, 2016. 46: pp. 139-144

[6] Magna, G., et al., Identification of mammography anomalies for breast cancer detection by an ensemble of classification models based on artificial immune system. Knowledge-Based Systems, 2016. 101: pp. 60-70

[7] Wang, S. and L. Wu, A novel method for magnetic resonance brain image classification based on adaptive chaotic PSO. Progress in Electromagnetics Research, 2010. 109: pp. 325-343

[8] Du, S., Multi-objective path finding in stochastic networks using a biogeography-based optimization method. Simulation, 2016. 92(7): pp. 637-647

[9] Sun, P., Pathological brain detection based on wavelet entropy and Hu moment invariants. BioMedical Materials and Engineering, 2015. 26(s1): pp. 1283-1290

[10]Zhou, X.-X., Comparison of machine learning methods for stationary wavelet entropy-based multiple sclerosis detection: decision tree, k-nearest neighbors, and support vector machine. Simulation, 2016. 92(9): pp. 861-871

[11]Lu, H.M., Facial Emotion Recognition Based on Biorthogonal Wavelet Entropy, Fuzzy Support Vector Machine, and Stratified Cross Validation. IEEE Access, 2016. 4: pp. 8375-8385 
[12]Nayak, D.R. Detection of unilateral hearing loss by Stationary Wavelet Entropy. CNS \& Neurological Disorders - Drug Targets, 2017. 16, DOI: 10.2174/1871527315666161026115046 (Online).

[13] Gorriz, J.M. and J. Ramírez, Wavelet entropy and directed acyclic graph support vector machine for detection of patients with unilateral hearing loss in MRI scanning. Frontiers in Computational Neuroscience, 2016. 10, Article ID: 160

[14]Lu, Z., A Pathological Brain Detection System Based on Radial Basis Function Neural Network. Journal of Medical Imaging and Health Informatics, 2016. 6(5): pp. 1218-1222

[15] Wei, L., Fruit classification by wavelet-entropy and feedforward neural network trained by fitness-scaled chaotic ABC and biogeography-based optimization. Entropy, 2015. 17(8): pp. 5711-5728

[16] Wu, J., Fruit classification by biogeography-based optimization and feedforward neural network. Expert Systems, 2016. 33(3): pp. 239-253

[17] Wang, S.-H. Single slice based detection for Alzheimer's disease via wavelet entropy and multilayer perceptron trained by biogeography-based optimization. Multimedia Tools and Applications, 2016, DOI: 10.1007/s11042-016-4222-4 (Online).

[18] Yang, J.F. and P. Sun, Magnetic resonance brain classification by a novel binary particle swarm optimization with mutation and time-varying acceleration coefficients. Biomedical EngineeringBiomedizinische Technik, 2016. 61(4): pp. 431-441

[19]Ji, G., A comprehensive survey on particle swarm optimization algorithm and its applications. Mathematical Problems in Engineering, 2015, Article ID: 931256

[20]Zhang, Y., Binary PSO with mutation operator for feature selection using decision tree applied to spam detection. Knowledge-Based Systems, 2014. 64: pp. 22-31

[21] Yang, G., Automated classification of brain images using wavelet-energy and biogeographybased optimization. Multimedia Tools and Applications, 2016. 75(23): pp. 15601-15617

[22] Jun, Y. and G. Wei, Find multi-objective paths in stochastic networks via chaotic immune PSO. Expert Systems with Applications, 2010. 37(3): pp. 1911-1919

[23]Phillips, P., et al., Pathological brain detection in magnetic resonance imaging scanning by wavelet entropy and hybridization of biogeography-based optimization and particle swarm optimization. Progress In Electromagnetics Research, 2015. 152: pp. 41-58

[24] Yang, S.N., et al., Identification of Breast Cancer Using Integrated Information from MRI and Mammography. Plos One, 2015. 10(6), Article ID: e0128404

[25] Milosevic, M., et al., Comparative analysis of breast cancer detection in mammograms and thermograms. Biomedical Engineering-Biomedizinische Technik, 2015. 60(1): pp. 49-56

[26]Lu, S., A note on the weight of inverse complexity in improved hybrid genetic algorithm. Journal of Medical Systems, 2016. 40(6), Article ID: 150

[27] Wei, L. and J. Yang, Fitness-scaling adaptive genetic algorithm with local search for solving the Multiple Depot Vehicle Routing Problem. SIMULATION, 2016. 92(7): pp. 601-616

[28]Zhang, Y., et al., UCAV path planning based on FSCABC. Information-An International Interdisciplinary Journal, 2011. 14(3): pp. 687-692

[29] Wu, L., Magnetic Resonance Brain Image Classification by an Improved Artificial Bee Colony Algorithm. Progress in Electromagnetics Research, 2011. 116: pp. 65-79

[30]Wu, L., Optimal multi-level Thresholding based on Maximum Tsallis Entropy via an Artificial Bee Colony Approach. Entropy, 2011. 13(4): pp. 841-859

[31]Zhang, Y., Stock market prediction of S\&P 500 via combination of improved BCO approach and BP neural network. Expert systems with applications, 2009. 36(5): pp. 8849-8854

[32] Wu, L., Weights optimization of neural network via improved BCO approach. Progress in Electromagnetics Research, 2008. 83: pp. 185-198

[33] Wu, L., Solving Two-Dimensional HP model by Firefly Algorithm and Simplified Energy Function. Mathematical Problems in Engineering, 2013, Article ID: 398141

[34]Liu, G., Pathological brain detection in MRI scanning by wavelet packet Tsallis entropy and fuzzy support vector machine. SpringerPlus, 2015. 4(1), Article ID: 716 
[35] Yang, J., Identification of green, Oolong and black teas in China via wavelet packet entropy and fuzzy support vector machine. Entropy, 2015. 17(10): pp. 6663-6682

[36] Yang, M., Dual-Tree Complex Wavelet Transform and Twin Support Vector Machine for Pathological Brain Detection. Applied Sciences, 2016. 6(6), Article ID: 169

[37]Chen, M., Morphological analysis of dendrites and spines by hybridization of ridge detection with twin support vector machine. PeerJ, 2016. 4, Article ID: e2207 